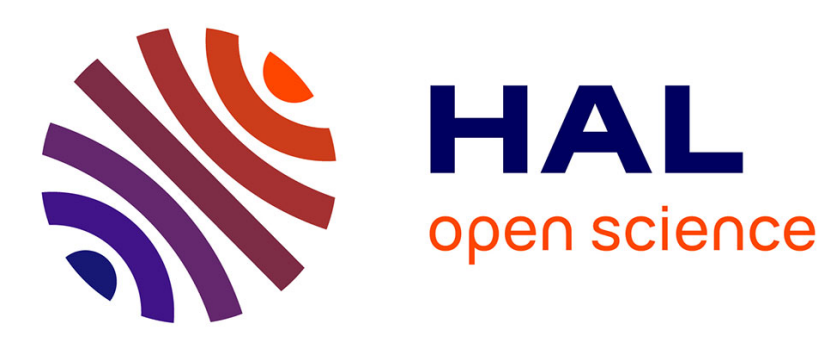

\title{
Diffusive Identification of Volterra Models by Cancellation of the Nonlinear Term
}

Céline Casenave, Gérard Montseny

\section{To cite this version:}

Céline Casenave, Gérard Montseny. Diffusive Identification of Volterra Models by Cancellation of the Nonlinear Term. 15th IFAC Symposium on System Identification, SYSID 2009, Jul 2009, Saint-Malo, France. pp.640-645, 10.3182/20090706-3-FR-2004.00106 . hal-01061517

\section{HAL Id: hal-01061517 https://hal.inria.fr/hal-01061517}

Submitted on 7 Sep 2014

HAL is a multi-disciplinary open access archive for the deposit and dissemination of scientific research documents, whether they are published or not. The documents may come from teaching and research institutions in France or abroad, or from public or private research centers.
L'archive ouverte pluridisciplinaire HAL, est destinée au dépôt et à la diffusion de documents scientifiques de niveau recherche, publiés ou non, émanant des établissements d'enseignement et de recherche français ou étrangers, des laboratoires publics ou privés. 


\title{
Diffusive Identification of Volterra Models by Cancellation of the Nonlinear Term
}

\author{
C. Casenave* , G. Montseny* \\ * LAAS-CNRS, Université de Toulouse \\ 7 av. du Colonel Roche, 31077 Toulouse cedex 4, France. \\ e-mail: casenave@laas.fr,montseny@laas.fr
}

\begin{abstract}
We present a new identification method for nonlinear Volterra models of the form $\mathcal{H} X=F(u, X)+v$ with $\mathcal{H}$ a causal convolution operator. It is based first on a suitable parameterization of $\mathcal{H}$ deduced from the so-called diffusive representation, devoted to state representations of integral operators, and second on a suitable operatorial transformation of the problem with the property that the nonlinear term $F(u, X)$ is cancelled, allowing to identify the operator $H\left(\partial_{t}\right)$ alone. Then, the nonlinear term can be identified from standard regression methods. For illustration, we implement this method on a concrete numerical example.
\end{abstract}

Keywords: Volterra Model, Nonlinear Model, Identification, Pseudo-inverse, Diffusive Representation, Operatorial Cancellation.

\section{INTRODUCTION}

We present an identification method for nonlinear Volterra models of the form:

$$
H\left(\partial_{t}\right) X=F(u, X)+v
$$

where $u(t), v(t), X(t) \in \mathbb{R}, t>0, F$ is a nonlinear function defined on $\mathbb{R}^{2}$ and $H\left(\partial_{t}\right)$ is a causal convolution operator defined on a suitable space of continuous functions with support in $\mathbb{R}_{t}^{+}$.

We suppose that problem (1) is well-posed in the sense of existence, uniqueness and continuous dependency on the inputs $u, v$ of the solution $X$. The operator $H\left(\partial_{t}\right)$ is also supposed invertible, so (1) can be rewritten under the form: $X(t)=\int_{0}^{t} k(t-s)[F(u(s), X(s))+v(s)] d s \forall t>0$, where $k$ is the impulse response of operator $H\left(\partial_{t}\right)^{-1}$.

Such models are frequently encountered in various domains:

- thermic phenomena (we will consider in section 4 the combustion model elaborated in Joulin (1985)),

- electrical engineering (Rumeau (2006)),

- linear SISO differential systems with nonlinear feedback:

$$
\left\{\begin{array}{l}
\dot{Z}=A Z+B F(u, X) \\
X=C Z,
\end{array}\right.
$$

with $H(p)^{-1}=C(p I-A)^{-1} B$,

- SISO partial differential systems on $\mathbb{R}_{t}^{+*} \times \Omega_{z} \subset \mathbb{R}^{n+1}$ of the form:

$$
\left\{\begin{array}{l}
\partial_{t} \varphi=A(z, \nabla) \varphi+F\left(u, \int T \varphi d z\right), \varphi_{0}=0 \\
X=\int T \varphi d z
\end{array}\right.
$$

which can be rewritten under the synthetic equation $\int_{0}^{t} k(t-s) F(u(s), X(s)) d s=X(t)$ where the function $k$ is the impulse response solution of $k=\int T \phi d z$, $\dot{\phi}=A(z, \nabla) \phi+\delta, \phi_{0}=0$.

- etc.
The identification problem under consideration in the sequel is to build estimations of $H\left(\partial_{t}\right)$ and $F$ from (noised) data $\tilde{X}=X+\eta$ where $\eta$ designates some additive measurement noise and $X$ is generated by an experimental process driven by known inputs $u, v$. The main difficulty when identifying such models results from the coupling between the dynamic operator $H\left(\partial_{t}\right)$ and the (static) function $F$ via equation (1). This difficulty is strengthened when operator $H\left(\partial_{t}\right)$ is non rational (which is in general the case), when $F$ is singular, when the system is unstable and/or when there exists some dynamic bifurcations (see section 4).

The proposed identification method is based first on a suitable parameterization of $H\left(\partial_{t}\right)$ deduced from the socalled diffusive representation (Montseny (2005)), devoted to state realizations of general integral causal operators. Following this approach, the complex dynamic nature of $H\left(\partial_{t}\right)$ is in some sense summarized by a few numerical parameters on which the identification problem will focus. Second, we introduce a suitable operatorial transformation of the problem with the property that the nonlinear term $F(u, X)$ is cancelled, allowing to identify the operator $H\left(\partial_{t}\right)$ alone from the available data. The nonlinear term can then be identified from the data completed by the knowledge of the previously identified $H\left(\partial_{t}\right)$.

This paper must be viewed as an extension of a previous one (Casenave (2008-2)) to which we broadly refer. We only present here the basic principles of the approach; more details, namely numerical questions, will be given in a future publication.

The paper is organized as follows. In section 2, we briefly present a simplified version of the diffusive representation. In section 3, we describe the proposed identification method in a general framework. In section 4, we finally implement this method on a concrete example, in order to highlight the relevance of the method. 


\section{DIFFUSIVE FORMULATION OF CAUSAL CONVOLUTION OPERATORS}

We only give a few basic notions; more details will be found in Casenave (2008-2). A complete statement of diffusive representation will be found in Montseny (2005). Various applications and questions relating to this approach will be found for example in Audounet (1998), Carmona (1998), Casenave (2007), Casenave (2008-1), Degerli (1999), Garcia (1998), Lenczner (2005), Levadoux (2003), Montseny (2007), Mouyon (2002), Rumeau (2006).

We consider a causal convolution operator defined, on any continuous function $u: \mathbb{R}^{+} \rightarrow \mathbb{R}$, by

$$
u \mapsto \int_{0}^{t} k(t-s) u(s) d s .
$$

We denote $K$ the Laplace transform of the (locally integrable) function $k$, and $K\left(\partial_{t}\right)$ the convolution operator defined by (2).

Let $u^{t}(s)=\mathbf{1}_{]-\infty, t]}(s) u(s)$ the restriction of $u$ to its past and $u_{t}(s)=u^{t}(t-s)$ the so-called "history" of $u$. From causality of $K\left(\partial_{t}\right)$, we deduce:

$$
\left[K\left(\partial_{t}\right)\left(u-u^{t}\right)\right](t)=0 \text { for all } t
$$

then, we have for any continuous function $u$ :

$$
\left[K\left(\partial_{t}\right) u\right](t)=\left[\mathcal{L}^{-1}(K \mathcal{L} u)\right](t)=\left[\mathcal{L}^{-1}\left(K \mathcal{L} u^{t}\right)\right](t) .
$$

We define:

$$
\Psi_{u}(t, p):=\mathrm{e}^{p t}\left(\mathcal{L} u^{t}\right)(p)=\left(\mathcal{L} u_{t}\right)(-p)
$$

by computing $\partial_{t} \mathcal{L} u_{t}$, Laplace inversion and use of (3), we have:

Lemma 1. 1. The function $\Psi_{u}$ is solution of the differential equation:

$$
\partial_{t} \Psi(t, p)=p \Psi(t, p)+u, \quad t>0, \quad \Psi(0, p)=0 .
$$

2. For any $b \geqslant 0$,

$$
\left[K\left(\partial_{t}\right) u\right](t)=\frac{1}{2 i \pi} \int_{b-i \infty}^{b+i \infty} K(p) \Psi_{u}(t, p) d p .
$$

We denote $\Omega$ the holomorphic domain of $K$ (after analytic continuation). Let $\gamma$ be a closed ${ }^{1}$ simple arc in $\mathbb{C}^{-}$; we denote $\Omega_{\gamma}^{+}$the exterior domain defined by $\gamma$, and $\Omega_{\gamma}^{-}$ the complementary of $\overline{\Omega_{\gamma}^{+}}$. By use of standard techniques (Cauchy theorem, Jordan lemma), it can be shown:

Lemma 2. For any $\gamma \subset \Omega$ such that $K$ is holomorphic in $\Omega_{\gamma}^{+}$, if $K(p) \rightarrow 0$ when $p \rightarrow \infty$ in $\Omega_{\gamma}^{+}$, then:

$$
\left[K\left(\partial_{t}\right) u\right](t)=\frac{1}{2 i \pi} \int_{\gamma} K(p) \Psi_{u}(t, p) d p .
$$

In the sequel, we use the convenient notation

$$
\langle\mu, \psi\rangle=\int \mu \psi d \xi
$$

Under hypothesis of lemma 2 (except $\gamma \subset \Omega$ ), we have (Montseny (2005)):

Theorem 3. If the possible singularities of $K$ on $\gamma$ are branching points such that $|K \circ \gamma|$ is locally integrable in their neighborhood, then, with $\mu=\frac{\gamma^{\prime}}{2 \mathrm{i} \pi} K \circ \gamma$ and

\footnotetext{
1 Possibly at infinity
}

$\psi_{u}(t,)=.\Psi_{u}(t,.) \circ \gamma:$

$$
\left[K\left(\partial_{t}\right) u\right](t)=\left\langle\mu, \psi_{u}(t, .)\right\rangle
$$

furthermore $\psi_{u}(t, \xi)$ is solution of the following evolution problem on $(t, \xi) \in \mathbb{R}^{*+} \times \mathbb{R}$ (of diffusive type):

$$
\partial_{t} \psi(t, \xi)=\gamma(\xi) \psi(t, \xi)+u(t), \psi(0, \xi)=0 .
$$

Definition 4. The function $\mu$ defined in theorem 3 is called $\gamma$-symbol of operator $K\left(\partial_{t}\right)$. The function $\psi_{u}$ solution of (8) is called the $\gamma$-representation or simply the diffusive representation of $u$.

Formulation $(8,7)$ can easily be extended to operators of the form $K\left(\partial_{t}\right) \circ \partial_{t}$ where $K\left(\partial_{t}\right)$ admits a $\gamma$-symbol. We have (formally):

$\left[K\left(\partial_{t}\right) \circ \partial_{t} u\right](t)=\left\langle\mu, \partial_{t} \psi_{u}(t,).\right\rangle=\left\langle\mu, \gamma \psi_{u}(t,)+.u(t)\right\rangle$,

with $\mu$ the $\gamma$-symbol of $K\left(\partial_{t}\right)$.

\section{IDENTIFICATION VIA OPERATORIAL CANCELLATION OF THE NONLINEAR TERM}

\subsection{The basic principle}

Given $E, F, G$ separable Hilbert spaces, $f: E \rightarrow G$ a nonlinear function and $A: F \rightarrow G$ linear, consider the abstract model:

$$
f(x)+A y+z=0,
$$

where $x, y, z$ are trajectories defined on $t \in[0, T]$ with values respectively in $E, F, G$. The problem under consideration is to estimate both $A$ and $f$ from (possibly) noised data trajectories $\tilde{y}, \tilde{x}, \tilde{z}$, that is to solve (in some sense):

$$
\min _{A, f}\|f(\tilde{x})+A \tilde{y}+\tilde{z}\|^{2},
$$

with $\|\cdot\|$ an hilbertian norm, for example $\int_{0}^{T}\|\cdot\|_{G}^{2} d t, \tilde{x}=$ $x+\varepsilon_{1}, \tilde{y}=y+\varepsilon_{2}, \tilde{z}=z+\varepsilon_{3}$ where $(x, y, z)$ is solution of (10) and $\varepsilon_{i}$ are some (sufficiently small) noises. Such a problem often presents some serious difficulties, in particular when $f$ is highly nonlinear (for example non differentiable, discontinuous or even weakly singular) and no a priori information about it is available. In such cases indeed, linear parametrizations ${ }^{2}$ of $f$ of the form $f=\sum_{i} a_{i} \mathbf{f}_{i}$ (with $\left(\mathbf{f}_{i}\right)$ a topological basis of $E$ chosen a priori) cannot be accurate enough except if the number of $a_{i}$ is quite large; but on the other hand, when the number of parameters to be estimated is excessive, estimation is generally bad due to excessive sensitivity to the measurement noises.

Now remark that thanks to linearity, operator $A$ could be easily estimated if the term $f(x)$ was known, the problem (11) then reducing to a standard least-square one:

$$
\min _{A}\|\mathcal{Y} \cdot A-b\|^{2}
$$

with solution formally given by the pseudo-inverse of operator $\mathcal{Y}$ (Ben-Israel (2003)):

$$
A^{*}=\mathcal{Y}^{\dagger} \cdot b \text {. }
$$

Following this remark, the method presented here-after allows to estimate $A$ alone from a suitable transformation of model (10) with the special property that the term $f(x)$ is

\footnotetext{
2 The advantage of which is to transform (10) into a linear problem.
} 
cancelled ${ }^{3}$ in a particular subset of equations specifically defined from the data $x$. Under some hypothesis frequently satisfied in practice, this "submodel" then reveals itself sufficient for a good estimation of $A$ under the form (12). Once operator $A$ is correctly estimated, it will then be easy to deduce estimations of $f$ from standard methods.

\subsection{The $f$-cancellation operator $\mathrm{D}_{x, \varepsilon}$}

We briefly introduce, in an abstract framework, the "difference" operator $\mathrm{D}_{x, \varepsilon}$ on which the proposed method is based.

Given $\mathbf{X}$ a Banach space and $\Omega$ a metric space, let denote by $C^{0}(\Omega ; \mathbf{X})$ the Banach space of continuous ${ }^{4}$ functions defined on $\Omega$ with values in $\mathbf{X}$, with norm $\|x\|=$ $\sup _{\xi \in \Omega}\|x(\xi)\|_{\mathbf{X}}$. Given $\mathbf{X}_{0} \subset \mathbf{X}$, let $C^{0}\left(\Omega ; \mathbf{X}_{0}\right):=\{x \in$ $\left.C^{0}(\Omega ; \mathbf{X}) ; \forall \xi \in \Omega, x(\xi) \in \mathbf{X}_{0}\right\}$. Given metric spaces ${ }^{5}$ $\Omega^{i}, i=1: n$, the norm on the product space $\prod_{i=1}^{n} C^{0}\left(\Omega^{i} ; \mathbf{X}\right)$ is defined by $\|x\|=\sup _{i} \sup _{\xi \in \Omega^{i}}\left\|x^{i}(\xi)\right\|_{\mathbf{x}}$. Finally, we denote $\Omega^{i, j}:=\Omega^{i} \times \Omega^{j}$ and the following operator $\mathrm{D}$ is defined:

$$
\begin{aligned}
\mathrm{D}: & \prod_{i=1}^{n} C^{0}\left(\Omega^{i} ; \mathbf{X}\right) \rightarrow \prod_{i, j=1}^{n} C^{0}\left(\Omega^{i, j} ; \mathbf{X}\right) \\
x=\left(x^{i}\right)_{i=1: n} & \mapsto \mathrm{D} x=\left(\mathrm{D}^{i, j} x\right)_{i, j=1: n},
\end{aligned}
$$

$\forall i, j \in\{1: n\}, \forall(t, \tau) \in \Omega^{i, j}, \quad\left(\mathrm{D}^{i, j} x\right)(t, \tau)=x^{i}(t)-x^{j}(\tau)$

The operator D is clearly linear. Thanks to the continuity of the + operation in Banach spaces, it is continuous; note that this last property is essential, namely for numerical treatments.

Let $\mathbf{Y}$ be another Banach space, $\mathbf{X}_{0} \subset \mathbf{X}$ and $f: \mathbf{X}_{0} \rightarrow$ $\mathbf{Y}$ continuous. For $x \in \prod_{i=1}^{n} C^{0}\left(\Omega^{i} ; \mathbf{X}_{0}\right)$, we denote by convention:

$$
f \circ x:=\left(f\left(x^{i}\right)\right)_{i=1: n} \in \prod_{i=1}^{n} C^{0}\left(\Omega^{i} ; \mathbf{Y}\right) .
$$

Note that if $f$ is linear, we have: $\mathrm{D}(f \circ x)=f \circ(\mathrm{D} x)$, this property being false in general.

Now consider the sets ${ }^{6}$ (see Figure 1):

$$
\Omega_{x, \varepsilon}^{i, j}:=\left\{(t, \tau) \in \Omega^{i, j} ;\left\|\left(\mathrm{D}^{i, j} x\right)(t, \tau)\right\| \leqslant \varepsilon\right\} ;
$$

for any $(i, j)$ s.t. $\Omega_{x, \varepsilon}^{i, j} \neq \varnothing$, we denote $\mathrm{D}_{x, \varepsilon}^{i, j}$ and $\mathrm{D}_{x, \varepsilon}$ the operators defined by

$$
\forall y, \mathrm{D}_{x, \varepsilon}^{i, j} y:=\left.\mathrm{D}^{i, j} y\right|_{\Omega_{x, \varepsilon}^{i, j}} \text { and } \mathrm{D}_{x, \varepsilon}:=\left(\mathrm{D}_{x, \varepsilon}^{i, j}\right)_{i, j} ;
$$

we have:

Proposition 1. For any $x \in \prod_{i=1}^{n} C^{0}\left(\Omega^{i} ; \mathbf{X}_{0}\right)$ and any continuous $f: \mathbf{X}_{0} \rightarrow \mathbf{Y}$, the following properties hold:

(1) $\mathrm{D}_{x, 0} x=0$

(2) $\mathrm{D}_{x, 0}(f \circ x)=0$

(3) $\left\|\mathrm{D}_{x, \varepsilon} x\right\| \leqslant \varepsilon$,

\footnotetext{
3 Up to a constant term: $f_{0}:=f\left(x\left(t_{0}\right)\right) \in G$ which will be identified simultaneously.

4 This hypothesis can be weakened by means of suitable technical adaptations.

5 In practice, $\Omega^{i}$ will be time intervals $\left[t_{0}^{i}, t_{f}^{i}\right]$.

6 The sets $\Omega_{x, \varepsilon}^{i, i}$ are never empty because $\left(\mathrm{D}^{i, i} x\right)(t, t)=0$. Furthermore, thanks to the continuity of $\mathrm{D} x$, we have $\bigcap_{\varepsilon>0} \Omega_{x, \varepsilon}^{i, j}=\Omega_{x, 0}^{i, j}$.
}

(4) $\forall\left(t_{\varepsilon}, \tau_{\varepsilon}\right) \in \Omega_{x, \varepsilon}^{i, j}$ s.t. $\left(t_{\varepsilon}, \tau_{\varepsilon}\right) \underset{\varepsilon \rightarrow 0}{\longrightarrow}(t, \tau) \in \Omega_{x, 0}^{i, j}$, $\lim _{\varepsilon \rightarrow 0}\left[\left(\mathrm{D}_{x, \varepsilon}^{i, j}(f \circ x)\right)\left(t_{\varepsilon}, \tau_{\varepsilon}\right)\right]=0$,

(5) If $f$ is $k$-Lipschitz, then $\left\|\mathrm{D}_{x, \varepsilon}(f \circ x)\right\| \leqslant k \varepsilon$.

Proof. (1) and (2) $\forall i, j \in\{1: n\}, \forall(t, \tau) \in \Omega_{x, 0}^{i, j}$, we have $\left(\mathrm{D}^{i, j} x\right)(t, \tau)=x^{i}(t)-x^{j}(\tau)=0$, that is $x^{i}(t)=x^{j}(\tau)$, and so $f\left(x^{i}(t)\right)=f\left(x^{j}(\tau)\right)$; then: $\left(\mathrm{D}^{i, j}(f \circ x)\right)(t, \tau)=f\left(x^{i}(t)\right)-$ $f\left(x^{j}(\tau)\right)=0$.

(3) is a direct consequence of the definition of $\Omega_{x, \varepsilon}^{i, j}$ and of the norm on $\prod_{i, j} C^{0}\left(\Omega_{x, \varepsilon}^{i, j} ; \mathbf{X}\right)$.

(4) First remark that $(t, \tau) \in \Omega_{x, 0}^{i, j} \Rightarrow x^{i}(t)=x^{j}(\tau)$; thanks to the continuity of $f$ and $x$, we then have: $\lim _{\varepsilon \rightarrow 0}\left(\mathrm{D}_{x, \varepsilon}^{i, j}(f \circ x)\right)\left(t_{\varepsilon}, \tau_{\varepsilon}\right)=\lim _{\varepsilon \rightarrow 0}\left(f\left(x^{i}\left(t_{\varepsilon}\right)\right)-f\left(x^{j}\left(\tau_{\varepsilon}\right)\right)\right)=$ $f\left(x^{i}(t)\right)-f\left(x^{j}(\tau)\right)=0$.

$$
\begin{array}{r}
(5)\left\|\mathrm{D}_{x, \varepsilon}(f \circ x)\right\|=\sup _{i, j} \sup _{\Omega_{x, \varepsilon}^{i, j}}\left\|f\left(x^{i}(t)\right)-f\left(x^{j}(\tau)\right)\right\| \\
\leqslant \sup _{i, j} \sup _{\Omega_{x, \varepsilon}^{i, j}} k\left\|x^{i}(t)-x^{j}(\tau)\right\| \leqslant k \varepsilon .
\end{array}
$$

Remark 1. The estimate (5) is uniform, while (4) relates to the weaker topology of simple convergence. For this reason, it can be expected that when $f$ is not Lipschitz-continuous on $\mathbf{X}_{0}$, numerical treatments can be somewhat more delicate. However, in many concrete situations, function $f$ is Lipschitz-continuous on subsets $\mathbf{X}_{\eta} \subset \mathbf{X}_{0}$, in such a way that the property $\left\|\mathrm{D}_{x, \varepsilon}\left(\tilde{f}_{\eta} \circ x\right)\right\| \leqslant k_{\eta} \varepsilon$ holds for any $k_{\eta}$-Lipschitz-continuous function $\tilde{f}_{\eta}$ (defined on $\mathbf{X}_{0}$ ) such that $\tilde{f}_{\eta}\left|\mathbf{x}_{\eta}=f\right| \mathbf{x}_{\eta}$. We will see on a concrete example (with $\mathbf{X}=\mathbf{Y}=\mathbb{R}, \mathbf{X}_{0}=\mathbb{R}^{+*}, f=\ln , \mathbf{X}_{\eta}=[\eta,+\infty[$ ) that such a property (in addition to (4)) can be sufficient to get good identification results.

For convenience, we will denote in the sequel:

$$
\Omega_{x, \varepsilon}:=\bigcup_{i, j}\left(\{(i, j)\} \times \Omega_{x, \varepsilon}^{i, j}\right) ;
$$

this set will be called the $\varepsilon$-kernel of Dx. Roughly speaking, it can be viewed as the set of all the $(i, j, t, \tau)$ such that $\left\|x^{i}(t)-x^{j}(\tau)\right\| \leqslant \varepsilon$. So, for any $y \in \prod_{i=1}^{n} C^{0}\left(\Omega^{i} ; \mathbf{Y}\right)$, the function $\mathrm{D}_{x, \varepsilon} y$ is simply defined by:

$$
\begin{aligned}
\mathrm{D}_{x, \varepsilon} y: \quad \Omega_{x, \varepsilon} & \rightarrow \mathbf{Y} \\
(i, j, t, \tau) & \mapsto y^{i}(t)-y^{j}(\tau) .
\end{aligned}
$$

Note that for numerical purposes, we will mainly have to consider discrete subsets of $\Omega_{x, \varepsilon}$, with elements $\left(i, j, t_{k}, \tau_{l}\right) \sim$ $(i, j, k, l) \in \mathbb{N}^{4}$. Numerical questions (in particular the choice of $\varepsilon$ and the construction of a discrete set $\Omega_{x, \varepsilon}$ from a discrete-time trajectory $x$ ) will be studied in a further publication.

\subsection{The $\mathrm{D}_{x, \varepsilon}$-transformed identification problem}

Let us consider the model (10): $f \circ x+A y+z=0$, now with $(x, y, z)$ a possibly vector trajectory with values in $E^{n} \times F^{n} \times G^{n}$, with $x^{i}, y^{i}, z^{i}$ defined on $\Omega^{i}=\left[t_{0}^{i}, t_{f}^{i}\right]$. By applying operator $\mathrm{D}_{x, 0}$ to both members of this equation, we get $\mathrm{D}_{x, 0}(f \circ x)+A \mathrm{D}_{x, 0} y+\mathrm{D}_{x, 0} z=0$ and, thanks to property (2) of proposition $1, A \mathrm{D}_{x, 0} y+\mathrm{D}_{x, 0} z=0$; note that this last equation is linear with respect to the (unknown) operator $A$. However, due to the fact that 

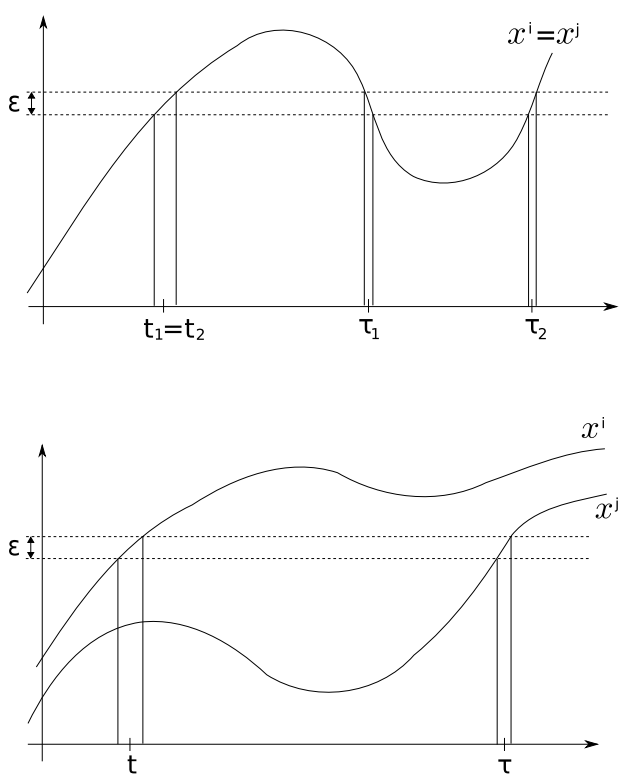

Fig. 1. Examples of $(t, \tau)$ in $\Omega_{x, \varepsilon}^{i, i}$ (top) and in $\Omega_{x, \varepsilon}^{i, j}$ (bottom)

operator $\mathrm{D}_{x, 0}$ is based on differences, equivalence is lost. To restore equivalence, it is necessary ${ }^{7}$ to consider an "initial" condition (at least one!) on (10): $f\left(x^{i}\left(t_{*}^{i}\right)\right)+$ $A y^{i}\left(t_{*}^{i}\right)+z^{i}\left(t_{*}^{i}\right)=0$ for some $t_{*}^{i} \in\left[t_{0}^{i}, t_{f}^{i}\right] ;$ so, with $n^{*} \leqslant n$ and by denoting $f_{0}:=\left(f\left(x^{i}\left(t_{*}^{i}\right)\right)\right) \in G^{n^{*}}, y_{0}:=\left(y^{i}\left(t_{*}^{i}\right)\right) \in$ $F^{n^{*}}, z_{0}:=\left(z^{i}\left(t_{*}^{i}\right)\right) \in G^{n^{*}}$, the model:

$$
\left\{\begin{array}{l}
A \mathrm{D}_{x, 0} y=-\mathrm{D}_{x, 0} z \\
f_{0}+A y_{0}=-z_{0}
\end{array}\right.
$$

can be equivalent to (10) (that is $(x, y, z)$ is solution of $(10)$ iff it is solution of (19)) if the set $\Omega_{x, 0}$ is large enough ${ }^{8}$. The interest of this new formulation is that both the unknowns $A$ and $f_{0}$ are linearly involved; formally, these unknowns can then be determined by means of the pseudoinverse of operator $\mathcal{Y}_{0}:\left(A, f_{0}\right) \mapsto\left(A \mathrm{D}_{x, 0} y, f_{0}+A y_{0}\right)$.

In general however, on the one hand the set $\Omega_{x, 0}$ may be too poor to get the strict equivalence of (19) and (10) and, on the other hand, the available data $\tilde{x}, \tilde{y}, \tilde{z}$ are noised. But in fact, it is only expected that some "good" identification of $\left(A, f_{0}\right)$ can be computed from the available data; so, thanks to properties (4), (5) of proposition 1 which allow to neglect (in some sense) the term $\mathrm{D}_{x, \varepsilon}(f \circ x)$, problem (19) is replaced by the weakened one, based on operator $\mathcal{Y}_{\varepsilon}:\left(A, f_{0}\right) \mapsto\left(A \mathrm{D}_{\tilde{x}, \varepsilon} \tilde{y}, f_{0}+A \tilde{y}_{0}\right)$ :

$$
\min _{\left(A, f_{0}\right)}\left\|\mathcal{Y}_{\varepsilon} \cdot\left(A, f_{0}\right)+\left(\mathrm{D}_{\tilde{x}, \varepsilon} \tilde{z}, \tilde{z}_{0}\right)\right\|^{2},
$$

with formal solution $\left(A^{*}, f_{0}^{*}\right)=-\mathcal{Y}_{\varepsilon}^{\dagger} \cdot\left(\mathrm{D}_{\tilde{x}, \varepsilon} \tilde{z}, \tilde{z}_{0}\right)$. If data $\tilde{x}, \tilde{y}, \tilde{z}$ allow to get $\left(A^{*}, f_{0}^{*}\right) \simeq\left(A, f_{0}\right)^{9}$, then we can expect from continuity properties:

$A^{*}\left(\tilde{y}^{i}(t)-\tilde{y}^{j}(\tau)\right)+\tilde{z}^{i}(t)-\tilde{z}^{j}(\tau) \simeq 0, \quad \forall(i, j, t, \tau) \in \Omega_{x, \varepsilon}$, and so, although $f$ is yet entirely unknown ${ }^{10}$ at this stage: $f\left(\tilde{x}^{i}\right)+A^{*} \tilde{y}^{i}+\tilde{z}^{i} \simeq 0$ in the sense $f\left(\tilde{x}^{i}(t)\right)+A^{*} \tilde{y}^{i}(t)+$ $\tilde{z}^{i}(t) \simeq 0, \forall t \in\left[t_{0}^{i}, t_{f}^{i}\right]$. Now, thanks to the property

\footnotetext{
7 But maybe not sufficient if data are not rich enough.

8 This should be clarified in a further work.

9 In particular if $n$ is sufficiently big and noises $\varepsilon_{i}$ are small enough.

This will be studied in a further work.

${ }^{10}$ Except its value at point $x_{0}=\left(x^{i}\left(t_{*}^{i}\right)\right) \in E^{n^{*}}$.
}

$f\left(\tilde{x}^{i}(t)\right) \simeq-A \tilde{y}^{i}(t)-\tilde{z}^{i}(t)$, identification of the nonlinear function $f$ can be achieved by means of classical regression methods implemented on the following "pseudo graph" of $f$ deduced from the available data:

$$
\mathcal{G}_{f}=\bigcup_{i=\{1: n\}, t \in\left[t_{0}^{i}, t_{f}^{i}\right]}\left\{\left(\tilde{x}^{i}(t),-A^{*} \tilde{y}^{i}(t)-\tilde{z}^{i}(t)\right)\right\} \subset E \times G \text {. }
$$

\subsection{Application to Volterra models under diffusive formulation}

We start from the diffusive formulation of model (1). Given the following diffusive realization of operator $H\left(\partial_{t}\right)$ :

$$
H\left(\partial_{t}\right) X=\left\langle\mu, \gamma \psi_{X}+X\right\rangle,
$$

with $\psi_{X}$ the diffusive representation of $X$, and $\mu$ the $\gamma$ symbol associated with operator $H\left(\partial_{t}\right) \circ \partial_{t}^{-1}$, the model (1) is equivalently rewritten:

$$
\left\langle\mu, \gamma \psi_{X}+X\right\rangle-F \circ(u, X)-v=0 .
$$

Thanks to the static nature of the linear operator $\varphi \mapsto$ $\langle\mu, \varphi\rangle$, that is $\forall t,(\langle\mu, \varphi\rangle)(t)=\langle\mu, \varphi(t)\rangle,(22)$ can be rewritten (in opposite to (1) which is a $t$-nonlocal model): $\forall i, t, \quad\left\langle\mu, \gamma \psi_{X^{i}}(t)+X^{i}(t)\right\rangle-F\left(u^{i}(t), X^{i}(t)\right)-v^{i}(t)=0$.

Then, to apply the identification method presented above to model (22), we must take: $\Omega^{i}:=\left[0, t_{f}^{i}\right], x:=(u, X)$, $y:=\gamma \psi_{X}+X, z:=-v, f:=-F, A: y \mapsto\langle\mu, y\rangle$. Indeed, it can be verified that model (22) is then written under the form (10). In particular, the unknowns to be identified are $\left(\mu, f_{0}\right)$ and the operator $\mathcal{Y}_{\varepsilon}$ defined in the previous section is expressed by $\mathcal{Y}_{\varepsilon}:\left(\mu, f_{0}\right) \mapsto\left(\left\langle\mu, \mathrm{D}_{\tilde{x}, \varepsilon} \tilde{y}\right\rangle, f_{0}+\left\langle\mu, \tilde{y}_{0}\right\rangle\right)$.

Remark 2. Thanks to proposition 1, some prefiltering $Q\left(\partial_{t}\right)$ can be envisaged on the data ${ }^{11} X$ and $v$. Indeed, by applying operator $Q\left(\partial_{t}\right)$ to $(1)$, we get the equivalent model $H\left(\partial_{t}\right) \circ Q\left(\partial_{t}\right) X=Q\left(\partial_{t}\right) F(u, X)+Q\left(\partial_{t}\right) v$; if, in a suitable sense, $Q\left(\partial_{t}\right) F(u, X) \simeq F(u, X)$, then $\mathrm{D}_{x, 0} Q\left(\partial_{t}\right) F(u, X) \simeq \mathrm{D}_{x, 0} F(u, X)=0$.

\section{A CONCRETE EXAMPLE}

In this section, we illustrate the identification method introduced above by implementing it on data elaborated from numerical simulations of a complex dynamic phenomenon studied in Joulin (1985), Audounet (1998).

\subsection{The model under consideration}

In Joulin (1985), Joulin elaborated a Volterra model to describe, in suitable thermodynamic conditions, the evolution of a spherical flame initiated by a source at point 0 in a mixture of reactive species. Under some reasonable physical hypothesis, such a phenomenon can be described by a system of two partial differential equations relating to the temperature and the mass density of the mixture. By considering the reactive zone as a thin sheet located on a sphere with radius $x(t)$, Joulin has established that when the flame is developing in free space, $x$ is solution of the following nonlinear singular Abel-Volterra equation $^{12}(u(t)$ designates the source strength at time $t)$ :

$$
x(t) \int_{0}^{t} \frac{\dot{x}(s) d s}{\sqrt{\pi(t-s)}}=x(t) \ln x(t)+u(t) \quad \forall t>0,
$$

\footnotetext{
${ }^{11}$ The new prefiltered data are $v^{\prime}:=Q\left(\partial_{t}\right) v, X^{\prime}=Q\left(\partial_{t}\right) X$

${ }^{12}$ Here adimensional for simplicity.
} 
with the additional conditions: $x\left(0^{+}\right)=0, u \geqslant 0, x \geqslant 0$ (whose physical interpretation is obvious). By denoting $H\left(\partial_{t}\right)$ the convolution operator ${ }^{13} x \mapsto \int_{0}^{t} \frac{\dot{x}(s) d s}{\sqrt{\pi(t-s)}}$ and $F(u, x)+v:=\ln x+\frac{u}{x}$, (24) can be formally rewritten under the form (1).

It has been shown in Audounet (1998) that the evolution problem (24) is well-posed, that is the solution $x$ exists, is unique and depends continuously on $u$. In real conditions however, various perturbations are involved in the evolution of $x$ (due for example to the loss of spatial symmetries), and both the convolution operator $H\left(\partial_{t}\right)$ and the function $F$ will be more or less far from the ideal ones. So, an identification process can be justified if accuracy of the model is required.

We consider the problem of identification of (10) from (noised) data $(u, x)$ obtained from highly accurate numerical simulations of (24) (not described here, see Casenave (2008-1) for numerical method for such simulations).

\subsection{On the dynamic behavior of the flame radius}

It has been shown in Audounet (1998) that there exists a threshold relating to the power of the source $u$, beyond which the flame is developing whereas a quenching occurs below. In that sense, this evolution phenomenon is essentially unstable with two qualitatively different behaviors. Because of the hereditary nature of the problem, it is difficult to know the value of this threshold which must be evaluated on the basis of numerical simulations. In Casenave (2008-2), examples are given of flame either quenching or developing, when the source function is defined, as in Audounet (1998), by:

$$
u(t)=E t^{0.3}(1-t) \mathbf{1}_{[0,1]}(t) .
$$

The shortcomings of such sensitive dynamic behaviors are reinforced by the fact that the input $u$ is dynamically poor and $f$ is singular, which generates ill-conditioned identification problems. As a consequence, this model can be viewed as a significant test for the proposed identification method.

\subsection{Formulation of the identification problem}

For identification purposes, the flame model under consideration can be written under the form (1); by denoting:

$$
\begin{aligned}
f(x) & :=F(u, x), \quad z:=\frac{u}{x}, \quad y:=\gamma \psi_{x}+x, \\
A y & =\langle\mu, y\rangle, \mathbf{X}=\mathbf{Y}=\mathbb{R}, \mathbf{X}_{0}=\mathbb{R}^{+*},
\end{aligned}
$$

we can then apply the method described in section 3 . A detailed description of the practical implementation of this method will be given in a future paper.

\subsection{Numerical results and comments}

Recall that the problem mainly consists in identifying the diffusive symbol $\mu$ of operator $H\left(\partial_{t}\right) \circ \partial_{t}^{-1}$

\footnotetext{
${ }^{13}$ Note that in this ideal case, $H\left(\partial_{t}\right)=\partial_{t}^{\frac{1}{2}}$. Under weak hypothesis on $\gamma$, this operator admits a $\gamma$-symbol $\mu$ (see (Casenave (2008-2))).
}

from numerical experimental data. These data are composed of 9 trajectories $\left(u^{i}, \tilde{x}^{i}\right)_{i=1: 9}$ (see figure 2) associated with 9 sources $u^{i}$ of the form (25) with $E=$ $1.5,1.6,1.7,1.73,1.74,1.75,2.0,3.0$ and 5.0. The time step $\Delta t$ has been taken equal to $10^{-5}$ and the maximal final time is $t_{f}=6$. The measured trajectories are $\tilde{x}^{i}=x^{i}+\eta v^{i}$ where $v^{i}$ is a unity gaussian white noise and $\eta=10^{-2}$.

The function $\gamma$ has been chosen as $\gamma(\xi)=|\xi| e^{i \alpha \operatorname{sign}(\xi)}$ with $\alpha=\frac{160}{180} \pi$. The $\xi$-discretization has been defined from 130 values $\xi_{l}$ geometrically spaced to cover 5 frequency decades from $10^{-2}$ to $10^{5}$ (see Casenave (2008-2)).

In figure 3 , we can see the so-identified diffusive symbol $\mu^{*}$ associated with $H^{*}\left(\partial_{t}\right) \circ \partial_{t}^{-1}$. The associated frequency response $H^{*}(i \omega)$ is given in figure 4 . In the covered frequency band, this frequency response is correctly identified. The results are close to the ones obtained in Casenave (2008-2) in the case where function $f$ was known.

As an example, two of the resulting pseudo graphs of $f$, namely (see section 3.3):

$$
\mathcal{G}_{f}^{i}=\bigcup_{t_{k}}\left\{\left(\tilde{x}^{i}\left(t_{k}\right),-A^{*} \tilde{y}^{i}\left(t_{k}\right)-\tilde{z}^{i}\left(t_{k}\right)\right)\right\}
$$

are visible in figure 5 . The function $f=\ln$ is correctly reconstructed. Note that although the function $f$ is not Lipschitz-continuous around $x=0$, property (5) of proposition 1 remains satisfied around this point.

Finally, identification of function $f$ has been achieved by means of a standard least-square method implemented on the whole available pseudo graph, given by (21); the result is shown in figure 6 . In spite of the poverty of the data, in particular at high frequencies, the identification remains good.

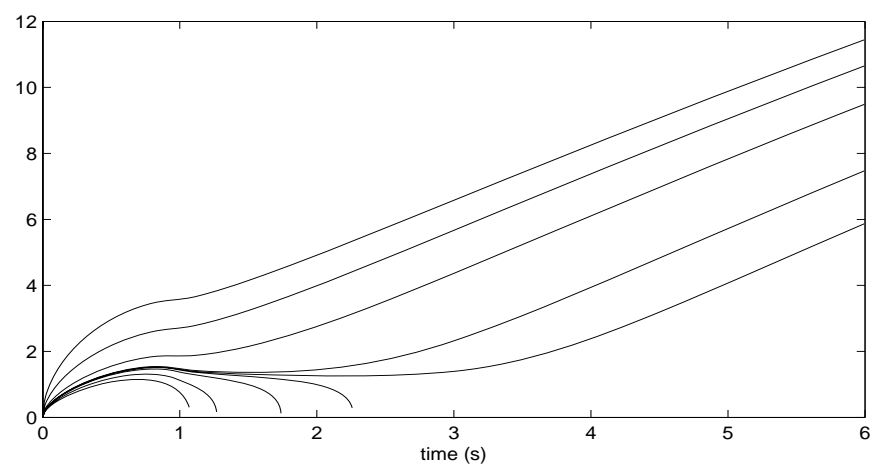

Fig. 2. The trajectories $x$ used for identification.

\section{REFERENCES}

J. Audounet, J.-M. Roquejoffre, An asymptotic fractional differential model of spherical flame, European Series on Applied and Industrial Mathematics (ESAIM): Proceedings, Vol.5, pp 15-28, Dec. 1998.

A. Ben-Israel, T.N.E. Greville, Generalized inverses: theory and applications, Springer-Verlag, New York, USA, 2003.

Y. Degerli, F. Lavernhe, P. Magnan, J. Farré, Bandlimited $1 / f^{\alpha}$ noise source, Electronics Letters, Vol. 35, no. 7, pp. 521-522, April 1999.

Ph. Carmona, L. Coutin, Fractional Brownian Motion and the Markov Property, Electronic Communications in Probability, Vol. 3, pp 95-105, Oct. 1998. 


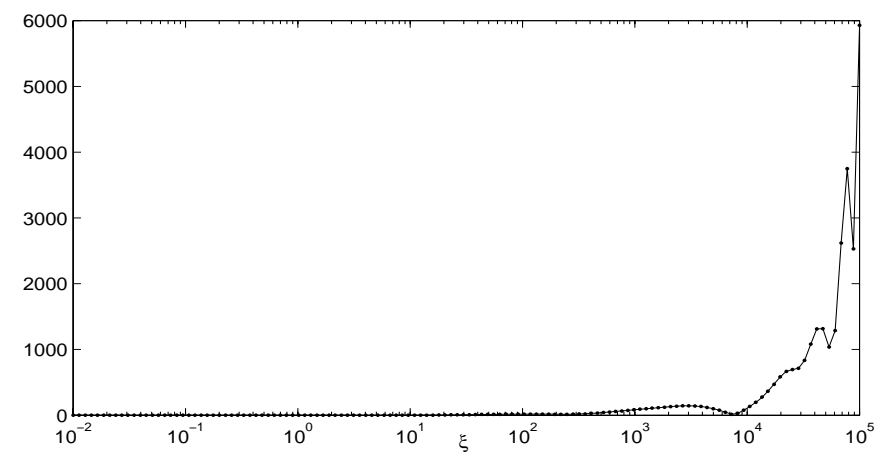

Fig. 3. The identified diffusive symbol $\mu^{*}\left(\xi_{l}\right)$.

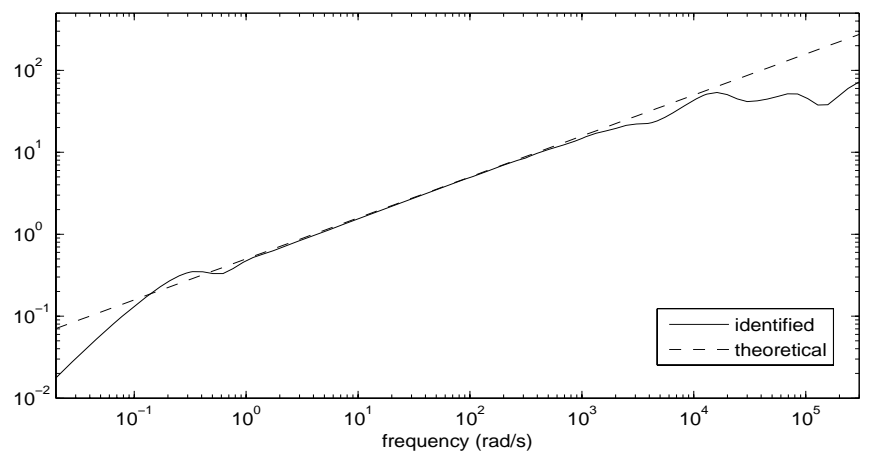

Fig. 4. Frequency response of the identified $H(i \omega)$.
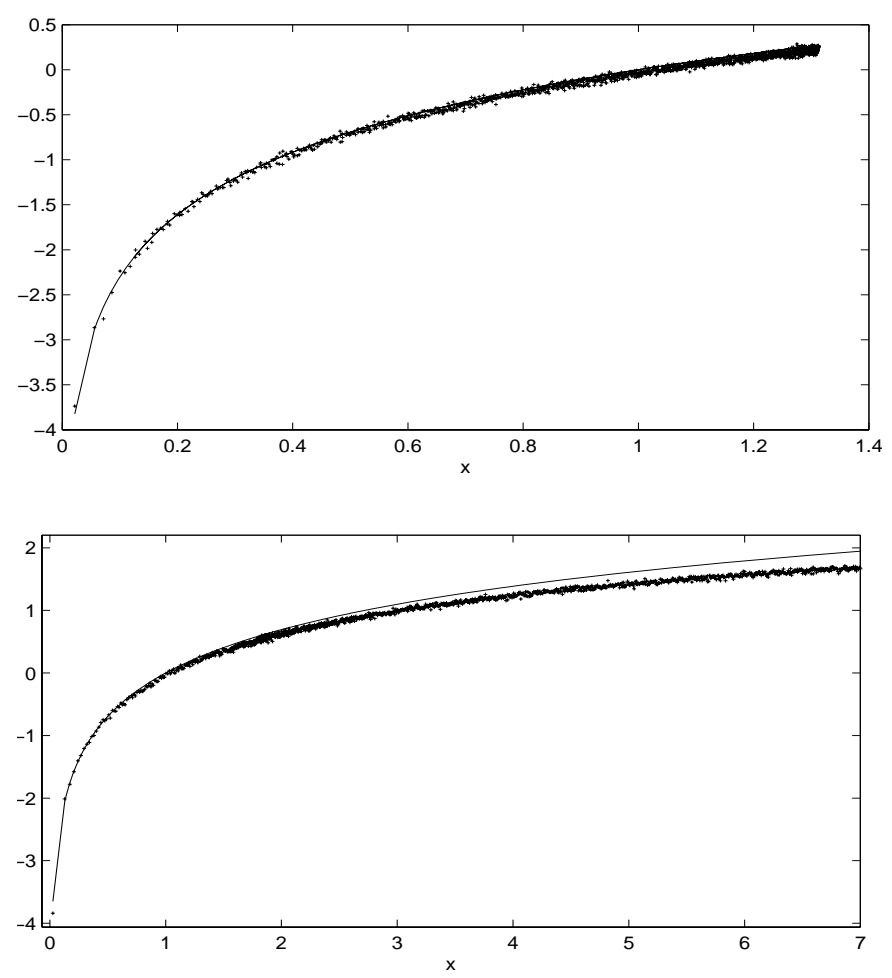

Fig. 5. Two of the pseudo grapfs $\mathcal{G}_{f}^{i}$.

C. Casenave, E. Montseny, Time-local dissipative formulation and stable numerical schemes for a class of integrodifferential wave equations, SIAM Journal on Applied Mathematics, Vol.68, N6, pp.1763-1783, 2008.

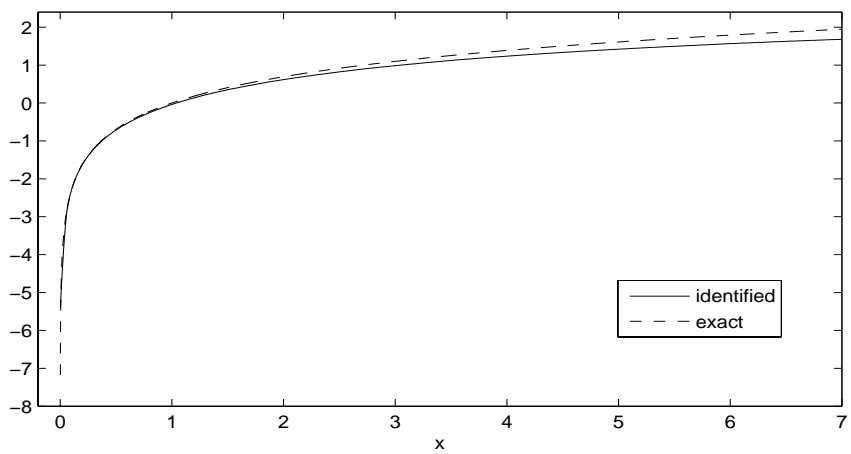

Fig. 6. Identified $f^{*}$.

C. Casenave, G. Montseny, Identification of Nonlinear Volterra Models by means of Diffusive Representation, 17th IFAC World Congress, Seoul (Korea), July 6-11, 2008, pp. 4024-4029.

C. Casenave, G. Montseny, Optimal identification of delay-diffusive operators and application to the acoustic impedance of absorbent materials, IFAC Workshop on Time Delay Systems, Nantes, Sept. 17-19, 2007.

G. Garcia, J. Bernussou, Identification of the dynamics of a lead acid battery by a diffusive model, European Series on Applied and Industrial Mathematics (ESAIM): Proceedings, Vol.5, pp 87-98, Dec. 1998.

G. Joulin, Point-source initiation of lean spherical flames of light reactants: an asymptotic theory, Comb. Sci. and Tech., pp 99-113, 1985.

M. Lenczner, G. Montseny, Diffusive realization of operators solutions of certain operational partial differential equations, Comptes Rendus de l'Académie des Sciences (Math.), Vol. 341 - No 12 - pp 737-740, Dec. 2005.

D. Levadoux, G. Montseny, Diffusive formulation of the impedance operator on circular boundary for $2 D$ wave equation, The Sixth International Conference on Mathematical and Numerical Aspects of Wave Propagation, June 30 - july 4, 2003, Jyväskylä, Finland.

G. Montseny, Diffusive representation for operators involving delays, in "Applications of time-delay systems", J.-J. Loiseau \& J. Chiasson eds. pp 217-232, SpringerVerlag, 2007.

G. Montseny, Représentation Diffusive, Hermes-Science, Paris, France, 2005.

G. Montseny, Simple approach to approximation and $d y$ namical realization of pseudodifferential time-operators such as fractional ones, IEEE Trans on Circ. \& Syst. II, Vol 51, No 11, pp 613-618, Nov. 2004.

G. Montseny, Diffusive representation of pseudodifferential time-operators, European Series on Applied and Industrial Mathematics (ESAIM): Proceedings, Vol.5, pp 159175, Dec. 1998.

Ph. Mouyon, N. Imbert, Identification of a $2 D$ turbulent wind spectrum, Aerospace Science and Technology, Vol 6, No 8, pp 599-605, Dec. 2002.

A. Rumeau, P. Bidan, T. Lebey, L. Marchin, B. Barbier, S. Guillemet, Behavior modeling of a $\mathrm{CaCu}_{3} \mathrm{Ti}_{4} \mathrm{O}_{12}$ ceramic for capacitor applications, IEEE Conference on Electrical Insulation and Dielectric Phenomena, Kansas City (Missouri USA), Oct. 15-18, 2006. 